\title{
The Dual Positive Actions of Colonic Release of Ibuprofen in TNBS-Induced Colitis Wistar Rats
}

\author{
Heni Rachmawati*, Anastasia, I Ketut Adnyana, Kusnandar Anggadiredja \\ School of Pharmacy Bandung Institute of Technology, Bandung, Indonesia \\ Email: ${ }^{*} h$ rachmawati@fa.itb.ac.id
}

Received 17 April 2014; revised 29 May 2014; accepted 16 June 2014

Copyright (C) 2014 by authors and Scientific Research Publishing Inc.

This work is licensed under the Creative Commons Attribution International License (CC BY). http://creativecommons.org/licenses/by/4.0/

c) (i) Open Access

\section{Abstract}

Ibuprofen is a relatively safe anti inflammatory drug among other NSAIDs. However, frequent and long-term administration of ibuprofen in conventional oral preparation is still considered for ulceration. As previously reported, the daily administration of the ibuprofen orally for 14 days in rats caused the gastroduodenal ulcer. Several mechanisms have been reported: the suppression of the gastric prostaglandin synthesis and the local irritant effect on epithelium due to the direct contact of drug with mucosal wall. In this work, developed ibuprofen pellet with double coatings aimed to release ibuprofen only when reaching colonic compartment. The results of pharmacokinetic study reported previously suggested that this might be a successful target. Present study described the potential benefits of this formula in exhibiting effective local anti inflammatory action in colon. Male Wistar rats were induced for ulcerative colitis with 2,4,6-trinitrobenzene sulfonic acid. Twenty four hours after induction, treatments were given using either ibuprofen suspension or pellet for 14 days. Ulcerations were observed visually, with gross anatomic and microscopic examinations. Group treated with ibuprofen pellet showed best recovery nearly close to healthy group. Moreover, the group did not develop ulceration in upper part of GIT. Colonic targeted ibuprofen pellet showed most effective local antiinflammatory action and at the same time reduced the ulcer formation in the upper part of GIT.

\section{Keywords}

Colonic Release, Ibuprofen, TNBS-Induced Colitis, Gastrointestinal Ulcer, Non-Steroid Anti-Inflammatory Drugs, Topical Irritation

\footnotetext{
*Corresponding author.
} 


\section{Introduction}

Nonsteroidal anti-inflammatory drugs (NSAIDs) including ibuprofen are commonly used to treat several disorders like pain, inflammation and fever. However, their use is associated with a relative high incidence of adverse effects in the gastrointestinal (GI) tract [1]-[3]. Such damages can form mucosal erosions or ulcers and can occur anywhere along the digestive system from the esophagus to the colon. In the small intestine, the damage can sometimes be found in long-term NSAID users. The greatest concern from a clinical standpoint is the progression of ulcers to the stage of the perforation and the risk of the severe bleeding from ulcers [1]. The ability of NSAIDs like ibuprofen to cause ulceration and bleeding in the upper gastrointestinal tract was first documented by the endoscopic study of Douthwaite and Lintott in 1938 [1].

The development of safer NSAIDs or effective therapies for the prevention of the adverse reactions of existing NSAIDs requires a better understanding of the pathogenesis of NSAID-induced ulcer disease. NSAIDs cause damage to the gastroduodenal mucosa via several mechanisms including the topical irritant effect of these drugs on the epithelium, impairment of the barrier properties of the mucosa, suppression of gastric prostaglandin synthesis, reduction of gastric mucosal blood flow and interference with the repair of superfacial injury [4]-[10]. The presence of acid in the lumen of the stomach also contributes to the pathogenesis of NSAID-induced ulcers and bleeding by impairing the regeneration process interfering with haemostasis and inactivating several growth factors that are critical in the mucosal defense and repair.

The main factor that limits NSAID in clinic is the progression of upper gastrointestinal adverse effects including ulcers, complications such as bleeding, and dyspepsia. Strategies had been recommended to decrease GI injury in NSAID users include co-therapy with misoprostol or proton-pump inhibitors and/or use of COX-2 selective inhibitors [11] [12].

In addition, modification of release of these NSAIDs in safer compartment of GI tract by formulation approach can be helpful somehow. We have developed the colonic release of the ibuprofen pellet by double layers coating of the pellet using inulin and shellac as the microbial-inner layer and the $\mathrm{pH}$-outer layer, respectively, providing a $\mathrm{pH}$ and microbial-controlled system. Other approaches have been reported to obtain colonic release included $\mathrm{pH}$ responsive and time responsive systems [13]-[15]. However, the appropriate performance of $\mathrm{pH}$ responsive system could not be guaranteed at different physiological conditions or disease states like ulcerative colitis with lower colonic pH. Similarly, time responsive system is limited when a subject encounters different gastric emptying and intestinal or colonic transit times.

Considering the lack of systems in subject variability, our formula was developed by combining $\mathrm{pH}$ and microbial responsive system using shellac and inulin. The aim was to obtain dual actions: reducing colonic inflammation and preventing gastroduodenal ulcer due to the direct contact of ibuprofen to the upper part of GI tract [16]. The pharmacokinetic of this formulation also confirmed the in vitro delay release but with unchanged bioavailability of ibuprofen. This result indicates that ibuprofen in the colon is absorbed in the same quantity as in gastric. Ibuprofen or iso-butyl-propanoic-phenolic acid is a relatively weak acid (pka 4.4) used as an anti-inflammatory agent. The low solubility in water or at acid $\mathrm{pH}$ results in the relatively long residence time in the stomach, further slowing down the absorption of the drug [17]. The conventional oral preparation of ibuprofen therefore enables to epithelial damage, especially at sites of direct contact with the GI mucosa [18]-[20].

In this study, we evaluated the previously developed colonic release system of ibuprofen formula to treat colonic inflammation. Colonic inflammation (colitis) in rat was induced by 2,4,6-trinitrobenzene sulfonic acid (TNBS). Many reports do not recommend the conventional oral dosage form of ibuprofen to treat this disease due to its ability to cause gastric bleeding and form ulceration in the gastric lining. Therefore, in addition to evaluate the potential action in the colonic inflammation, this formula was also tested for its benefit to the prevention of the ulceration in the upper part of the GI tract. The latter is expected to be due to the avoidance of the direct contact of the ibuprofen with the mucosal wall. To confirm the potential gastroduodenal ulceration, ibuprofen suspension was used as a reference.

\section{Materials and Methods}

\subsection{Materials}

Ibuprofen (PT Kimia Farma, Bandung-Indonesia), mesalamine or 5-aminosalicylic acid (Salofalk ${ }^{\circledR}$, commercially purchased), 2.4.6 trinitrobenzene sulfonic acid (TNBS, Sigma, St. Louis, MO, USA). Sodium chloride, Sodium dihydrogen phosphate, Sodium hydroxide, ethanol (Merck, Darmstad, Germany), formalin (PT Brata- 
chem, Bandung-Indonesia), urethane. Suspension of reference drugs were freshly prepared using 0.5\% Carboxy methyl cellulose (PT Kimia Farma, Indonesia) in normal saline as a vehicle.

\subsection{Preparation of Double Coating of Ibuprofen Pellet}

Pellets were prepared as previously described [16]. Briefly, extrusion-spheronization was a technique used for pellet preparation. The core ibuprofen pellet was consisted of 33.33\% ibuprofen, $43 \%$ microcrystalline cellulose (Avicel1 PH101), 17.41\% guar gum, 0.97\% Sodium alginate, 0.97\% Calcium acetate, and 4\% Polyvinylpyrrolidone K-30. Further on, pellets which size of $560 \mu \mathrm{m}$ were coated subsequently with inulin and shellac as inner and film coatings, respectively. In both coating materials, Polyethylene glycol $6000(0.4 \%)$ was added as a plasticizer. Coating was performed using a fluidized bed coater (Jiangsu Jiafa Granulating drying equip-ment, Changzhou, China).

\subsection{Animal}

Male Wistar rats, age of 2 - 3 months with the body weight in the range of 200 - 250 g were used in this study. The rats received a standard diet and were housed under standard laboratory conditions. The study protocol was approved by the Local Ethic Committee for Care and Use of Laboratory Animals (number 300/FKUP-RSHS/ KEPK/Kep./EC/2009. December 29, 2009). The study was also performed according to international guidelines on animal experimentation.

\subsection{Colonic Inflammation (Colitis) Induction}

The 2,4,6-trinitrobenzene sulfonic acid (TNBS)-induced rat colitis represents an experimental model for human Inflammatory Bowel Disease (IBD). In this model, rats receive an enema containing TNBS in ethanol. TNBS will break the mucosal barrier and allow penetration of the substance into the bowel wall. This acute model rapidly induces colonic inflammation in a reproducible manner.

Ulcerative colitis (UC) was induced according to the method of Morris et al. [21] with minor modifications. Briefly, before induction of colitis, rats were starved for $12 \mathrm{~h}$ but had free access to water. The rats were lightly anesthetized with urethane $25 \%$ through intraperitonial injection. A rubber catheter was inserted rectally into the colon with the tip approximately $8 \mathrm{~cm}$ proximal to the anus. TNBS (dissolved in ethanol 1:2 ratio) was instilled into the colon of rats as $80 \mathrm{mg} / \mathrm{kg} \mathrm{BB}$. Twenty four hours after induction, the rats were applied for experiment.

\subsection{Experimental Groups}

The rats were randomly divided into 5 groups ( $\mathrm{n}=10$ each) as described below:

A: Healthy control group, received only vehicle (distilled water)/day for 2 weeks.

B: Group of rats with TNBS-induced colitis, received only vehicle orally for 2 weeks.

C: Group of rats with TNBS-induced colitis, treated with ibuprofen suspension orally at a dose of $18 \mathrm{mg} / \mathrm{kg}$ body weight/day for 2 weeks.

D: Group of rats with TNBS-induced colitis, treated with ibuprofen pellet orally at a dose of $18 \mathrm{mg} / \mathrm{kg}$ body weight/day for 2 weeks.

E: Group of rats with TNBS-induced colitis, treated with mesalamine suspension orally at dose of $180 \mathrm{mg} / \mathrm{kg}$ body weight/day for 2 weeks.

During experiment, all rats were observed closely and characteristics of stool and body weight of each rat were recorded to assess the disease activity index (DAI) according to Vowinkel et al. [22]. DAI was determined by combining scores of weight loss, stool consistency, and bleeding. Each score was determined as follows, change in weight (0: $<1 \%, 1: 1 \%-5 \%, 2: 5 \%-10 \%, 4:>15 \%)$, stool blood (0: negative, 2: positive) or gross bleeding [4], and stool consistency (0: normal, 2: loose stools, 4: diarrhea) as previously described. These index represented the severity of macroscopically visible colonic damage was assessed by means of the scoring system shown in Table 1. At day 15 after induction, all rats were sacrificed and the stomach and colon were collected for biopsy and microscopic evaluations. Meanwhile, the intestines were collected only for macroscopic observation. This was then followed by cutting open their abdomen and examining the appearance of colon, intestine, and stomach. Thereafter, distal colon was removed. It was cut longitudinally and gently cleaned of fecal content using phosphate buffer saline. Wet weight (mg) of the distal colon, the length of colon and colon index were recorded for each specimen (Table 2). 
Table 1. The severity of macroscopically visible colonic damage reflected by disease activity index (DAI) of all groups during 14 days of observation.

\begin{tabular}{cccccc}
\hline Day & $\mathbf{A}$ & $\mathbf{B}$ & $\mathbf{C}$ & $\mathbf{D}$ & $\mathbf{E}$ \\
\hline $\mathbf{1}$ & $0^{\mathrm{a}}$ & $6.67 \pm 0.82$ & $6.33 \pm 0.52$ & $5.50 \pm 0.55$ & $5.80 \pm 1.48$ \\
$\mathbf{2}$ & $0^{\mathrm{a}}$ & $5.33 \pm 0.52$ & $5.50 \pm 1.05$ & $5.00 \pm 1.26$ & $4.40 \pm 1.67$ \\
$\mathbf{3}$ & $0^{\mathrm{a}}$ & $4.33 \pm 1.63$ & $5.50 \pm 0.84$ & $4.50 \pm 0.84$ & $3.40 \pm 0.89$ \\
$\mathbf{4}$ & $0^{\mathrm{a}}$ & $4.67 \pm 1.21$ & $4.83 \pm 1.47$ & $4.00 \pm 1.09$ & $3.60 \pm 1.52$ \\
$\mathbf{5}$ & $0^{\mathrm{a}}$ & $4.00 \pm 1.26$ & $4.67 \pm 1.75$ & $3.50 \pm 1.05$ & $3.20 \pm 2.39$ \\
$\mathbf{6}$ & $0^{\mathrm{a}}$ & $3.17 \pm 1.60$ & $4.50 \pm 1.22$ & $3.00 \pm 0.63$ & $3.40 \pm 2.19$ \\
$\mathbf{7}$ & $0^{\mathrm{a}}$ & $2.83 \pm 0.98$ & $4.50 \pm 1.87^{\mathrm{b}}$ & $2.50 \pm 1.05$ & $3.20 \pm 1.64$ \\
$\mathbf{8}$ & $0^{\mathrm{a}}$ & $2.50 \pm 0.84$ & $3.17 \pm 1.33^{\mathrm{b}}$ & $1.67 \pm 1.50$ & $2.20 \pm 1.09$ \\
$\mathbf{9}$ & $0^{\mathrm{a}}$ & $2.33 \pm 1.03$ & $3.17 \pm 1.33^{\mathrm{b}}$ & $1.33 \pm 1.03$ & $2.00 \pm 0.71$ \\
$\mathbf{1 0}$ & $0^{\mathrm{a}}$ & $2.50 \pm 0.84$ & $3.17 \pm 1.47^{\mathrm{b}}$ & $1.67 \pm 0.82$ & $2.40 \pm 0.55$ \\
$\mathbf{1 1}$ & $0^{\mathrm{a}}$ & $2.00 \pm 0.63$ & $2.67 \pm 1.03^{\mathrm{b}}$ & $0.67 \pm 0.82$ & $2.80 \pm 1.30^{\mathrm{b}}$ \\
$\mathbf{1 2}$ & $0^{\mathrm{a}}$ & $2.17 \pm 0.41$ & $3.00 \pm 1.79^{\mathrm{b}}$ & $0.83 \pm 0.75^{\mathrm{a}}$ & $2.00 \pm 0.71$ \\
$\mathbf{1 3}$ & $0^{\mathrm{a}}$ & $2.50 \pm 1.05$ & $2.50 \pm 0.55^{\mathrm{b}}$ & $0.50 \pm 0.55^{\mathrm{a}}$ & $1.20 \pm 0.45^{\mathrm{a}}$ \\
$\mathbf{1 4}$ & $0^{\mathrm{a}}$ & 2.00 & $1.33 \pm 0.52^{\mathrm{a} . \mathrm{b}}$ & $0.50 \pm 0.55^{\mathrm{a}}$ & $1.17 \pm 0.89^{\mathrm{a} . \mathrm{b}}$ \\
\hline
\end{tabular}

$\mathrm{a}=$ significantly different as compared to control group $(\mathrm{P}<0.05)$. $\mathrm{b}=$ significantly different as compared to group $\mathrm{D}(\mathrm{P}<0.05)$. All groups at all times were significantly different than the normal animals $(\mathrm{P}<0.05)$. A: Healthy control group; B: Group of rats with TNBS-induced colitis; C: Group of rats with TNBS-induced colitis, treated with ibuprofen suspension; D: Group of rats with TNBS-induced colitis, treated with ibuprofen pellet; E: Group of rats with TNBS-induced colitis, treated with mesalamine suspension.

Table 2. Criteria applied to the macroscopic injuries in the rat colon of all groups.

\begin{tabular}{cccc}
\hline Groups & Colon length & Colon weight & Colon index (\%) \\
\hline A & $12.85 \pm 0.65$ & $1.86 \pm 0.12^{\mathrm{a}}$ & $0.67 \pm 0.03^{\mathrm{a}}$ \\
B & $12.48 \pm 1.49$ & $2.91 \pm 0.91$ & $1.39 \pm 0.34$ \\
C & $12.74 \pm 1.67$ & $1.87 \pm 0.12^{\mathrm{a}}$ & $0.92 \pm 0.05^{\mathrm{a}}$ \\
D & $12.66 \pm 1.04$ & $1.82 \pm 0.17^{\mathrm{a}}$ & $0.82 \pm 0.07^{\mathrm{a}}$ \\
E & $13.29 \pm 1.42$ & $2.12 \pm 0.46^{\mathrm{a}}$ & $0.83 \pm 0.07^{\mathrm{a}}$ \\
\hline
\end{tabular}

$\mathrm{a}$ = significantly different as compared to untreated TNBS-induced colitis (group B) $(\mathrm{P}<0.05)$. A: Healthy control group; B: Group of rats with TNBS-induced colitis; C: Group of rats with TNBS-induced colitis, treated with ibuprofen suspension; D: Group of rats with TNBS-induced colitis, treated with ibuprofen pellet; E: Group of rats with TNBS-induced colitis, treated with mesalamine suspension.

\subsection{Histopathological Examination}

Colon and stomach tissues were individually fixed in $10 \%$ formalin, dehydrated, paraffin embedded, processed, sectioned in slices with $4 \mu \mathrm{m}$ thickness and stained with $\mathrm{H} \& \mathrm{E}$ (haematoxylin and eosin). The microscopic and histological scoring was carried out by a pathologist, blind to the treated groups.

\subsection{Statistical Analysis}

Data were expressed as mean \pm standard deviation, with statistical significance analyzed by unpaired t-test. The significance was considered at $\mathrm{P}$ values $<0.05$.

\section{Results}

\subsection{Disease Activity Index (DAI)}

Twenty four hours after administration of TNBS, all groups induced by TNBS began to show increased in DAI indicated by symptoms such as obvious diarrhea, inactivity and anorexia, while there were no symptoms in Group A. DAI of all rats differed significantly at different times. Following treatment, DAI began to decrease, especially group treated with ibuprofen pellet (D). Already after 8 days treatment, the DAI in this group was 
nearly close to normal group. In contrast, the DAI in group C and E remained higher even at last day of treatments (Table 1).

\subsection{Macroscopic Observation}

Swelling, edema, hyperemia, and ulcer were seen on the colonic mucosal surface of rats in Group B represented by higher weight and index colon (Table 2). In contrast, these damages were relieved in Group D, which was treated with ibuprofen pellet. In comparison to Group A, the weight and colon index of Group B, and C was significantly increased $(P<0.05)$. However, treated with either ibuprofen pellet (D) or mesalamine (E), the parameters decreased significantly ( $\mathrm{P}<0.05$ ), as compared to untreated Group B. This observation was also confirmed by Figure 1 agreeing with results as shown in Table 2.

In all groups, the adipose tissues surrounding intestine and colon were found. However, untreated control group showed more adipose tissues which were tightly attached to the intestine and colon (Figure 2(c)) as compared to healthy as well as treated groups. Treatment either with ibuprofen pellet and mesalamine reduced significantly the number of adipose tissues (Figure 2(a)). In contrast, administration of ibuprofen suspension did not diminish the number of adipose tissue (Figure 2(b)).
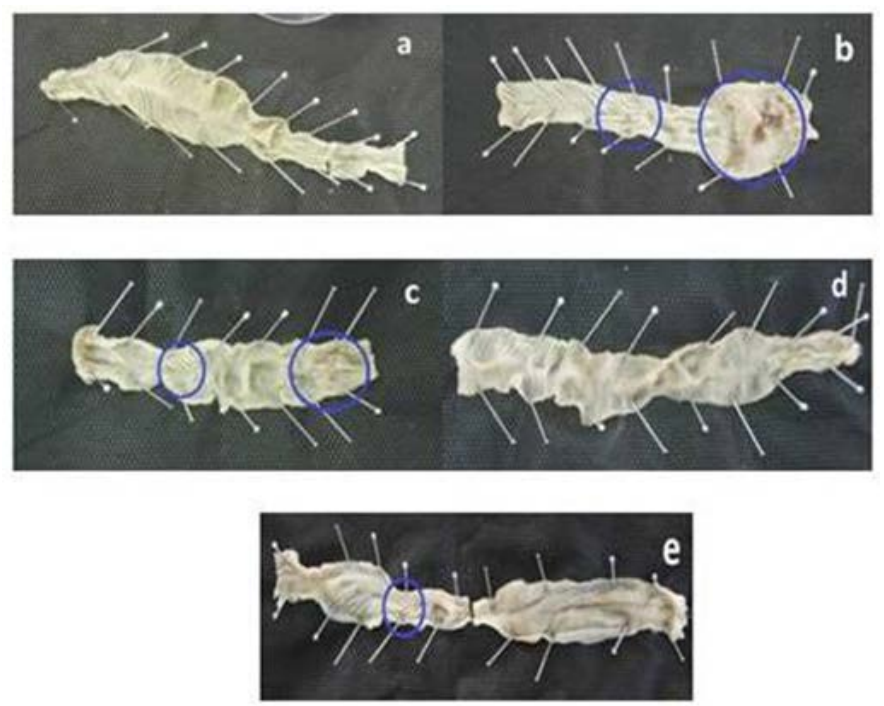

Figure 1. Macroscopic presentation of colon after 14 days of experiment. (a) Normal; (b) Untreated TNBS-induced group; (c) TNBSinduced group treated with suspensi ibuprofen; (d) TNBS-induced group treated with ibuprofen pellet; (e) TNBS-induced group treated with mesalamine. The large circle showed the colon damage, while small circle showed the thickening of colon wall.

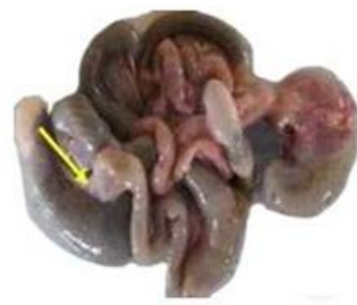

(a)

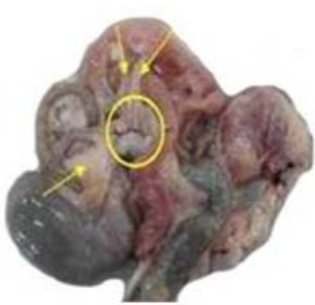

(b)

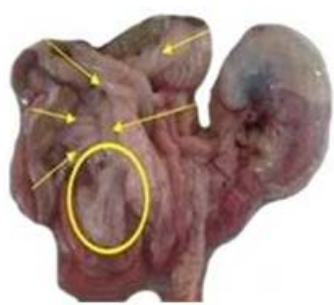

(c)

Figure 2. Macroscopic presentation of intestine and colon indicated adhesions. (a) Treated with ibuprofen pellet and mesalamine; (b) Treated with ibuprofen suspension; (c) Untreated group. Arrows and circles show the adipose tissue binding to the intestine. The adhesion level was proportional to the number of adipose tissues present in the TNBS-induced groups. 
Particularly in untreated group, the intestine adhesion occured due to improved mesenteric capillary permeability leading to exudation of fibrin [23].

\subsection{Macroscopic Changes of Gastric}

Figure 3 shows the macroscopic presentation of stomach in all groups. As seen, only group given ibuprofen suspension developed ulcer indicated by formation of reddish lesion on the gastric wall (Figure 3(c)).

\subsection{Histopathologic Changes of Colons}

In untreated Group B, histopathologic assessment revealed the most severe colonic inflammation with abundant infiltrated inflammatory cells in the colon, predominantly the mucosa and submucosa, or abscess (Figures 4(b1)-(b3)). The colonic structure of normal rat was shown to be well-organized with no damage observed. The goblet cells in colonic mucosa were intact with no sign of cell infiltration in the sub-mucosae. The circled area in Figures 4(b.1)-(b.3), 4(c.1)-(c.3), and 4(e.1) showed damaged, disintegrated goblet cells of varying sizes. Figure 2(b) also depicts the damaged colonic mucosa. Damage to or loss of goblet cells as well as disintegration of colonic mucosa may interfere with water and electrolytes absorption leading to poor fecal consistency (as shown by DAI observation). Colonic histological examination in control and ibuprofen-treated group revealed massive infiltration of inflammatory cells into colonic submucosa (boxed area in Figures 4(b.1) and 4(c.3)). This might be responsible for the visible thickening of colonic wall and the enlargement of colonic segment (indication of inflammatory response). The goblet cells in the colon of ibuprofen pellet- or mesalamine-treated rats were found to be well-organized as seen in normal animals. Infiltration of inflammatory cells was not observed in the ibuprofen pellet group, but occured at low degree in the mesalamine group (Figure 4(e.1)) that caused colonic thickening.

\subsection{Histopathologic Changes of Stomach}

Results of gastric histological examination showed that the lamina propria of normal group was well-organized and tend to be solid. In control group, the lamina propria was seen thinner but well-organized. On the other hand, the lamina propria of ibuprofen suspension group had lesion in several areas, the cells were not well-organized (looked less solid). Meanwhile, gastric histological presentation of ibuprofen pellet and mesalamine groups was the same as that in normal group (Figure 5).
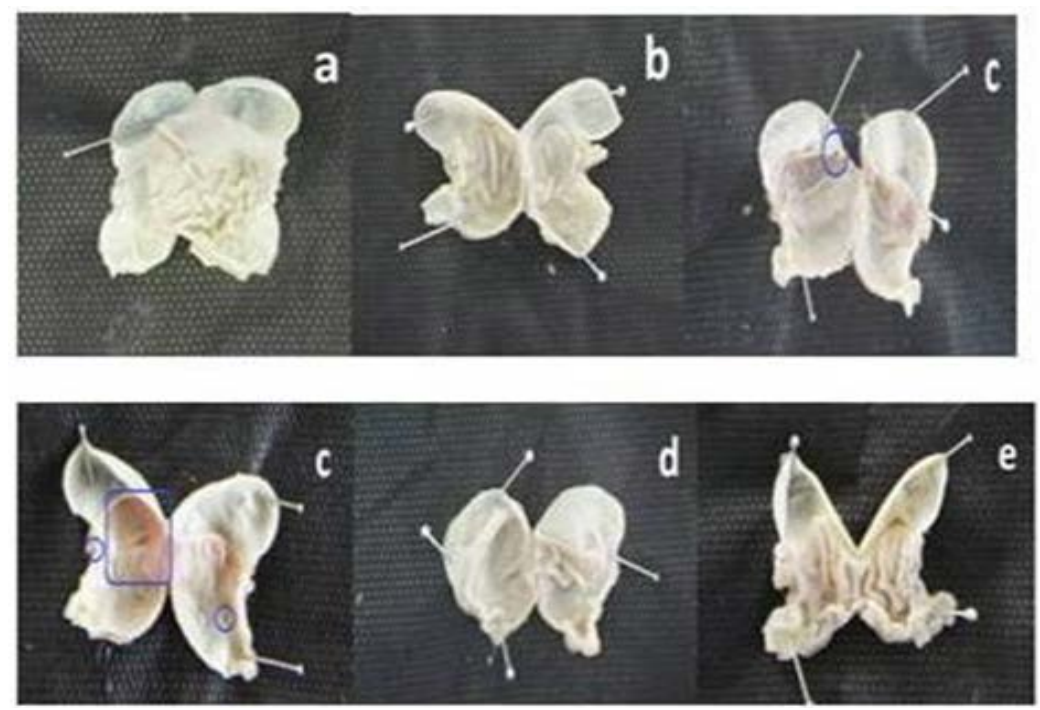

Figure 3. Macroscopic presentation of stomach. (a) Normal group; (b) Untreated TNBS-induced group; (c) TNBS-induced group treated with ibuprofen suspension; (d) TNBS-induced group treated with ibuprofen pellet; (e) TNBS-induced group treated with mesalamine suspension. Only group $\mathrm{C}$ showed gastric ulcer (blue marks). 

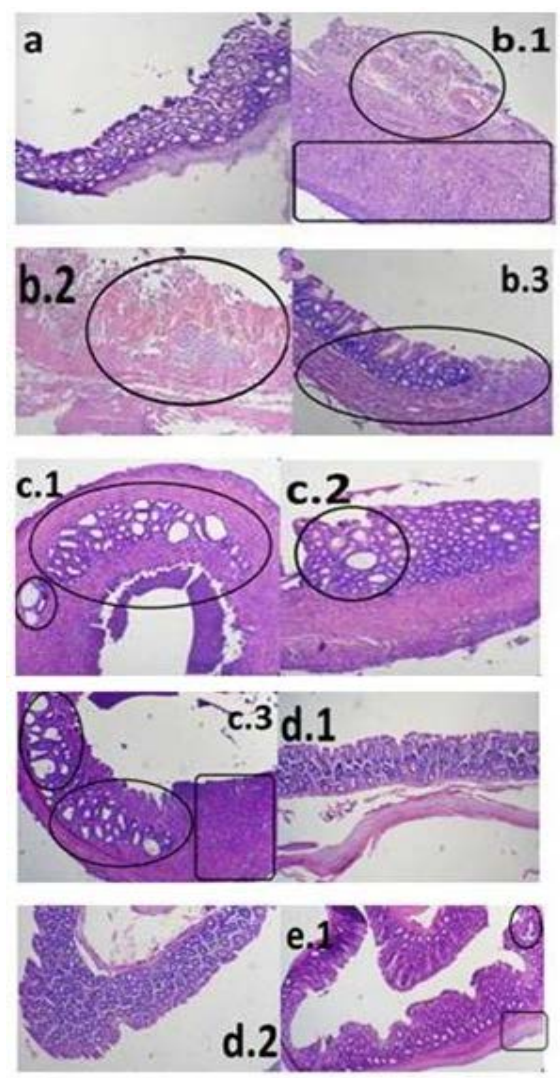

Figure 4. Microscopic presentation of colon by hematoxylin-eosin staining (HE). (a) Normal group; (b) Untreated TNBS-induced group; (c) TNBS-induced group treated with ibuprofen suspension; (d) TNBS-induced group treated with ibuprofen pellet; (e) TNBSinduced group treated with mesalamine suspension. It was shown the damage in goblet cells (signed by circle) and infiltration of inflammatory cells (signed by box).

\section{Discussion}

Ibuprofen causes damage in the upper gastrointestinal (GI) tract by impairing the ability of the mucosa to resist and respond to injury. The mechanisms responsible for ibuprofen-induced ulcerative lesions of the GI tract are not completely understood, particularly with respect to the lesions in the small intestine. A number of possible etiological factors have been proposed, including direct toxic effects of this drug on the epithelium, alterations in the mucosal microcirculation and disturbance of normal repair process. Ibuprofen is weak acid that produces epithelial damage at sites of contact with the GI mucosa. Although ibuprofen is known as relatively safe NSAIDs to cause GIT ulcer, one must be aware when using the long-term oral administration of this drug in the conventional dosage form, especially because of topical irritation on the mucosa. This was confirmed in this study, shown by group treated with ibuprofen suspension. Obvious direct contact of ibuprofen on GI wall was seen with the presence of stomach and intestine damages. Another mechanism by which ibuprofen and other NSAIDs damage the gastroduodenal epithelium is via the uncoupling of oxidative phosphorylation in the epithelial cells [24] [25]. Various NSAIDs have been shown to uncouple mitochondrial respiration, leading to a depletion of ATP and therefore a reduced ability to regulate normal cellular functions, such as the maintenance of intracellular $\mathrm{pH}$. Therefore, avoiding the direct contact of ibuprofen as reported in this study, reduced the GIT ulcer development. Ibuprofen pellet we developed only releases ibuprofen in the colonic compartment as described in 

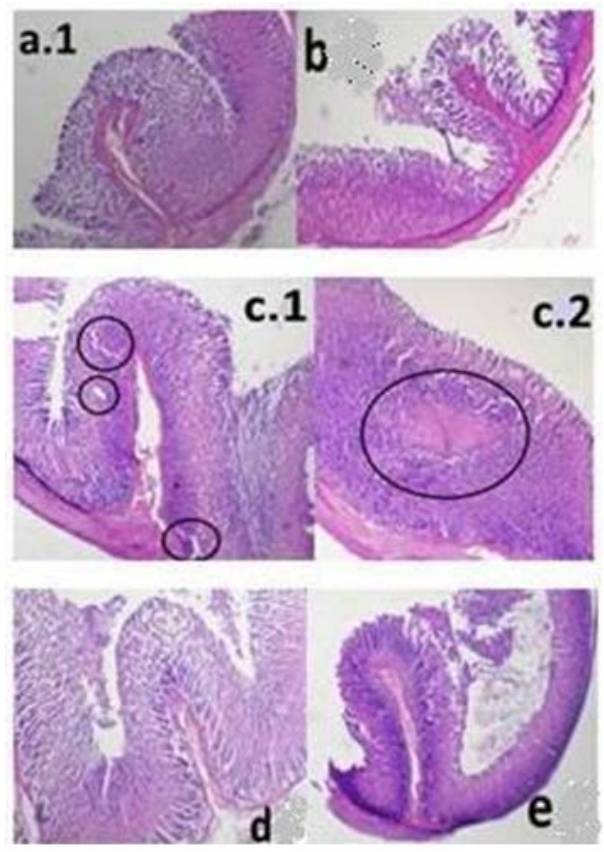

Figure 5. Histological examinations of gastric mucosal tissue by hematoxylin and eosin stain. Ulcer formation with distorted gastric glands, a damaged mucosal epithelium and cell debris are shown in c (ibuprofen suspension); however, administration of ibuprofen pellet and mesalammin protected against these changes, as shown in d (ibuprofen pellet) and e (mesalamine). In group treated with ibuprofen suspension, the damage in lamina propia was highlighted (c1, circle), while the presence of lesion in lamina propia was indicated (c2, circle). Magnification 100×.

our previous work [16]. Inulin, a bacterially degradable polysaccharide, used as an outer coating prevented the release of ibuprofen from pellet in the stomach as well as in intestine [26]. The use of specifically sensitive polymer to colonic environment may provide better selectivity for drug release in the large intestine. Due to high site-specificity of this polysaccharide and their sensitivity to degradation by colonic enzymes, more effective anti-inflammatory action of ibuprofen was achieved. Meanwhile, the contribution of outer layer of shellac and polymeric system in the pellet formulation were aimed to control premature swelling and dissolution of polysaccharides. Shellac is a natural material and soluble only in basic environment. The ability of shellac layer to prevent the release of ibuprofen from pellet can be explained by the fact that after introduction into an intestinal lumen ( $\mathrm{pH}$ 6.8), there was a slow solubilization. This led to the situation in which channels within the film through which ibuprofen diffused was formed slowly (3 - 4 hours) [16].

TNBS-induced rat colitis represents an experimental model for human Inflammatory Bowel Disease (IBD) [27] [28]. Histologically, colonic inflammation includes mucosal and submucosal infiltration by polymorphonuclear leukocytes, macrophages, lymphocytes, connective tissue mast cells, and fibroblasts. Segmental ulceration and inflammation were common within 3 weeks after induction of colitis [25]. In our study, the 40 rats that intrarectally given TNBS dissolved in ethanol at the dose of $25 \mathrm{mg} / \mathrm{rat}$ developed such characteristics as somatic pathological signs, mucosal lesions and colonic histopathological changes. From 10 to $24 \mathrm{~h}$ after the intrarectal dose of TNBS, all rats (in Groups B, C, D, E) developed diarrhea with varied severity, and some of the rats excreted bloody stool (Table 1). The presence of intestinal adhesion and tissues adiposity as shown in Figure 1 was obviously linked to the progression of inflammation. Both $\mathrm{T}_{\mathrm{H} 1}$ and $\mathrm{T}_{\mathrm{H} 17}$ cells and associated cytokines supposed to play a role in the underlying inflammatory responses. The inflamed colon showed increased weight and 
thickness, especially in the distal part as presented in Table 2 and Figure 1(b). TNBS-induced inflammation is associated primarily with increased inflammatory cellular infiltration, irregular crypts and loss of Goblet cells as seen in Figure 4. All of these findings indicated that our induction protocol was successful to develop ulcerative colitis model, allowing us to compare the effects of different treatments.

As of the second day, clinic DAI was seemed indistinguishable among these 40 rats in Groups B, C, D and E. As the treatment continued, however, DAI in treated Groups D and E decreased on the fourth day, while in untreated Group B and the group treated with ibuprofen suspension (C), DAI decreased slowly after sixth day. Animal treated with ibuprofen suspension turned out to have worst DAI as compared to untreated group. This is indicative of the efficacy of treatment with ibuprofen pellet or mesalamine. Judging from these results, it was suggested that reduction of TNBS-induced inflammatory symptoms by either ibuprofen or mesalamine was achieved through similar mechanism. Ibuprofen as well as mesalamine may work by blocking the activity of cyclooxygenase and lipoxygenase, the two enzymes which are over-expressed during ulcerative colitis with the consequent increased of prostaglandins production [24] [25]. During the course of treatment, the severity of DAI in all groups remained in the order of $\mathrm{B}>=\mathrm{C}>\mathrm{E}>\mathrm{D}>\mathrm{A}$, suggested the superiority of the efficacy of treatment with colonic release ibuprofen or mesalamine. Surprisingly, treatment with ibuprofen released in the colonic compartment showed the most effective treatment even as compared to oral suspension of mesalamine, a standard drug for ulcerative colitis. In addition to DAI, the macroscopic as well as histological observation confirmed the benefit effect of ibuprofen released in the colonic compartment. This finding might be explained due to the direct contact of ibuprofen on the inflamed tissue. Thus, colonic targeted delivery of ibuprofen showed better local effect as compared to conventional (suspension) oral route of mesalamine.

Macroscopic as well as microscopic evaluations on upper part of GI tract in particular in the gastric segment confirmed the effectiveness of colonic targeting of ibuprofen (Figure 2, Figure 3 and Figure 5). Symptoms of GI tract ulceration were not observed in group treated with this preparation. This result clearly explains the benefit of avoiding direct contact of ibuprofen with the upper part of GI tract. Meanwhile, the disappearance of GI tract ulceration in group treated with oral suspension of mesalamine confirmed other reports that as an amino-salicylate derivative, mesalamine rarely causes gastric ulcer [29] [30].

\section{Conclusion}

Taken together, results of present study suggest that ibuprofen targeted to be released in the colonic compartment shows dual potential benefits, which are preventing the development of GI tract ulcer and at the same time relieving the consequences of inflammatory reaction. The use of a combination of shellac and inulin to coat ibuprofen pellet is very effective in controlling the release of ibuprofen after oral administration. Due to the high site-specificity of this polysaccharide and their sensitivity to degradation by colonic enzymes, more effective anti-inflammatory action of ibuprofen was achieved, even better when compared to conventional oral delivery of mesalamin as a standard drug for ulcerative colitis. In summary, oral colon-specific delivery system of ibuprofen is a valuable method for the treatment of colon-related diseases such as ulcerative colitis.

\section{Conflict of Interest}

We wish to confirm that there are no known conflicts of interest associated with this publication and there has been no significant financial support for this work that could have influenced its outcome.

\section{Acknowledgements}

This study was financially supported by ITB research grant Indonesia, contract number: 848/I1.C03/PM/2012.

\section{References}

[1] Douthwaite, A.H. and Lintott, S.A.M. (1983) Gastroscopic Observation of the Effect of Aspirin and Certain Other Substances on the Stomach. Lancet, 2, 1222-1225.

[2] Gabriel, S.E. and Bombardier, C. (1990) NSAID Induced Ulcers. An Emerging Epidemic? The Journal of Rheumatology, 17, 1-4.

[3] Sakuma, T., Gocho, S., Ogasawara, F., et al. (2012) A Case of Small Bowel Ulcer Caused by NSAIDs and Detected after Capsule Endoscope Retention. The Tokai Journal of Experimental and Clinical Medicine, 37, 14-18. 
[4] Fromm, D. (1987) How Do Non-Steroidal Anti-Inflammatory Drugs Affect Gastric Mucosal Defense? Clinical Investigative Medicine, 10, 251-258.

[5] Whittle, B.J.R. (1977) Mechanism Underlying Gastric Mucosal Damage Induced by Indomethacin and Bile Salts, and the Actions of Prostaglandins. British Journal of Pharmacology, 60, 455-460.

http://dx.doi.org/10.1111/j.1476-5381.1977.tb07522.x

[6] Valerie V. (2005) Drug-Induced Peptic Ulcer Disease. Journal of the Malta College of Pharmacy Practice, 10, 15-19.

[7] Matteo, F., Luca, A., Rocchina, C., Marco, T. and Corrado, B. (2011) Pathophysiology of Gastric Ulcer Development and Healing: Molecular Mechanisms and Novel Therapeutic Options. In Tech, 114-142.

[8] Lacy, E.R. (1987) Gastric Mucosal Defense after Super Facial Injury. Clinical Investigative Medicine, 10, $189-200$.

[9] IMS Heath (2004) National Prescription Audit plus ${ }^{\mathrm{TM}}$.

[10] Lanza, F.L. (1998) A Guideline for the Treatment and Prevention of NSAID-Induced Ulcers. The American Journal of Gastroenterology, 93, 2037-2046. http://dx.doi.org/10.1111/j.1572-0241.1998.00588.x

[11] Hippisley-Cox, J., Coupland, C. and Logan, R. (2005) Risk of Adverse Gastrointestinal Outcomes in Patients Taking Cyclo-Oxygenase-2 Inhibitors or Conventional Non-Steroidal Anti-Inflammatory Drugs: Population Based Nested Case-Control Analysis. British Medical Journal, 331, 1310-1316. http://dx.doi.org/10.1136/bmj.331.7528.1310

[12] Bombardier, C., Laine, L., Reicin, A., et al. (2000) Comparison of upper Gastrointestinal Toxicity of Rofecoxib and Naproxen in Patients with Rheumatoid Arthritis. VIGOR Study Group. The New England Journal of Medicine, 343, 1520-1528. http://dx.doi.org/10.1056/NEJM200011233432103

[13] Peter, J.W. and Lisbel, I. (1997) Colonic Drug Delivery. Drug Development and Industrial Pharmacy, 23, $893-913$.

[14] Mayur, M.P. (2011) Cutting-Edge Technologies in Colon-Targeted Drug Delivery Systems. Drug Development and Industrial Pharmacy, 8, 1245-1258.

[15] Huiming, L., Feng, Z., Wenmin, Y., et al. (2011) Development of Multiple-Unit Colon-Targeted Drug Delivery System by Using Alginate: In Vitro and In Vivo Evaluation. Drug Development and Industrial Pharmacy, 37, 1347-1356. http://dx.doi.org/10.3109/03639045.2011.575163

[16] Rachmawati, H., Mudhakir, D. and Kusuma, J. (2011) Combination of Inulin-Shellac as a Unique Coating Formulation for Design of Colonic Delivery Dosage Form of Ibuprofen. International Journal of Pharmaceutical Sciences and Research, 2, 1-7.

[17] Chiarini, A., Tartarini, A. and Fini, A. (1984) pH-Solubility Relationship and Partition Coefficients for Some Anti-Inflammatory Arylaliphatic Acids. Archiv der Pharmazie, 317, 268-273. http://dx.doi.org/10.1002/ardp.19843170314

[18] Koyama, R., Kataoka, H., Tanaka, Y., Nakatsugi, S. and Furukawa, M. (1999) Effect of Caffeine on Ibuprofen-Induced Gastric Mucosal Damage in Rats. Journal of Pharmacy and Pharmacology, 51, 817-824. http://dx.doi.org/10.1211/0022357991773014

[19] Lanza, F.L., Royer, G.L., Nelson, R.S., Rack, M.F., Seckman, C.E. and Schwartz, J.H. (1986) Effect of Acetaminophen on Human Gastric Mucosal Injury Caused by Ibuprofen. Gut, 27, 440-443. http://dx.doi.org/10.1136/gut.27.4.440

[20] Balasubramanian, T., Somasundaram, M. and Felix, A.J.W. (2004) Taurine Prevents Ibuprofen-Induced Gastric Mucosal Lesions and Influences Endogenous Antioxidant Status of Stomach in Rats. The Scientific World Journal, 4, 10461054. http://dx.doi.org/10.1100/tsw.2004.207

[21] Morris, G.P., Beck, P.L., Herridge, M.S., Depew, W.T., Szewczuk, M.R. and Wallace, J.L. (1989) Hapten-Induced Model of Chronic Inflammation and Ulceration in the Rat Colon. Gastroenterology, 96, 795-803.

[22] Vowinkel, T., Kalogeris, T.J., Mori, M., Christian, F., Krieglstein, D. and Granger, N. (2004) Impact of Dextran Sulfate Sodium Load on the Severity of Inflammation in Experimental Colitis. Digestive Diseases and Sciences, 49, 556564. http://dx.doi.org/10.1023/B:DDAS.0000026298.72088.f7

[23] Wakefield, A.J., Dhillon, A.P., Rowles, P.M., Sawyerr, A.M., Pittilo, R.M., Lewis, A.A.M. and Pounder, R.E. (1989) Pathogenesis of Crohn's Disease: Multifocal Gastrointestinal Infarction. The Lancet, 2, 1057-1062. http://dx.doi.org/10.1016/S0140-6736(89)91078-7

[24] Somasundaram, S., Hayllar, H., Rafi, S., Wrigglesworth, J.M., Macpherson, A.J.S. and Bjarnason, I. (1995) The Biochemical Basis of Non-Steroidal Anti Inflammatory Drug-Induced Damage to the Gastrointestinal Tract: A Review and a Hypothesis. Scandinavian Journal of Gastroenterology, 30, 289-299. http://dx.doi.org/10.3109/00365529509093280

[25] Mahmud, T., Wrigglesworth, J.M., Scott, D.L. and Bjarnason, I. (1996) Mitochondrial Function and Modification of NSAID Carboxyl Moiety. InflammoPharmacology, 4, 189-194. http://dx.doi.org/10.1007/BF02735474

[26] Vervoort L. and Kinget R. (1996) In Vitro Degradation by Colonic Bacteria on Inulin HP Incorporated in Eudragit RS Films. International Journal of Pharmaceutics, 129, 185-190. http://dx.doi.org/10.1016/0378-5173(95)04322-5 
[27] Motavallian-Naenin, A., Andalib, S., Rabbani, M., Mahzouni, P., Afsharipour, M. and Minaiyan, M. (2012) Validation and Optimization of Experimental Colitis Induction in Rats Using 2,4,6-Trinitrobenzene Sulfonic Acid. Archive of Research in Pharmaceutical Sciences, 7, 159-169.

[28] Krimsky, M., Yedgar, S., Aptekar, L., Schwob, O., Goshen, G., Gruzman, A., et al. (2003) Amelioration of TNBS-Induced Colon Inflammation in Rats by Phospholipase A 2 Inhibitor. American Journal of Physiology, 285, G586-G592.

[29] Love, B.L. and Miller, A.D. (2012) Extended-Release Mesalamine Granules for Ulcerative Colitis. Annals of Pharmacotherapy, 46, 1529-1536. http://dx.doi.org/10.1345/aph.1R171

[30] Ham, M. and Moss, A.C. (2012) Mesalamine in the Treatment and Maintenance of Remission of Ulcerative Colitis. Expert Review of Clinical Pharmacology, 5, 113-123. http://dx.doi.org/10.1586/ecp.12.2 
Scientific Research Publishing (SCIRP) is one of the largest Open Access journal publishers. It is currently publishing more than 200 open access, online, peer-reviewed journals covering a wide range of academic disciplines. SCIRP serves the worldwide academic communities and contributes to the progress and application of science with its publication.

Other selected journals from SCIRP are listed as below. Submit your manuscript to us via either submit@scirp.org or Online Submission Portal.
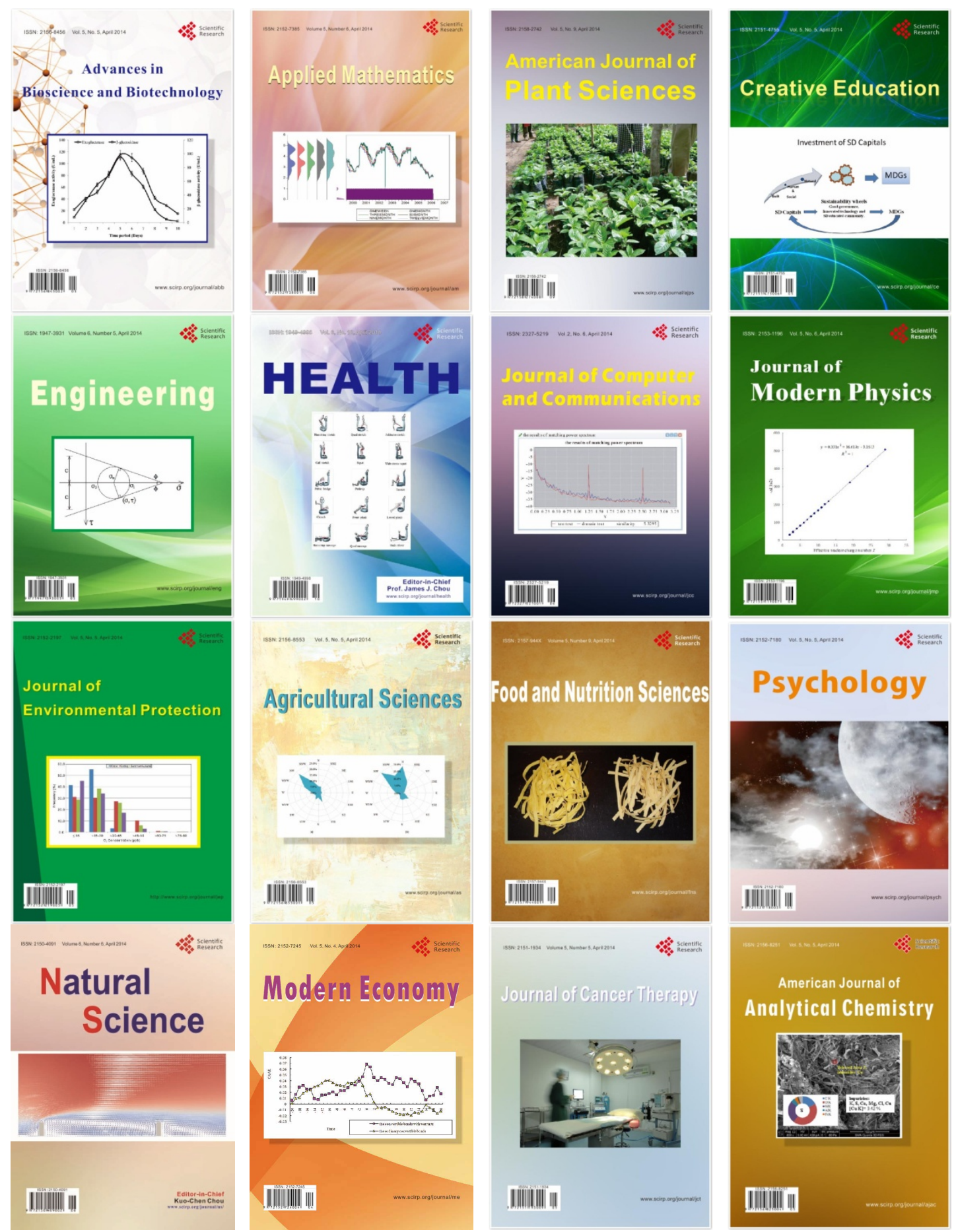